\title{
Data Mining for the Identification of Known and Candidate Transthyretin Amyloidosis Variants in the Saudi Population
}

\section{Mohamed Abouelhoda}

KFSH\&RC: King Faisal Specialist Hospital and Research Center

\section{Dania Mohty}

KFSH\&RC: King Faisal Specialist Hospital and Research Center

Islam Alayary

Phizer

\section{Brian F. Meyer}

KFSH\&RC: King Faisal Specialist Hospital and Research Center

\section{Stefan T. Arold}

KAUST: King Abdullah University of Science and Technology

\section{Bahaa M. Fadel}

KFSH\&RC: King Faisal Specialist Hospital and Research Center

Dorota Monies ( $\nabla$ moniesdm@gmail.com )

KFSHRC: King Faisal Specialist Hospital and Research Center https://orcid.org/0000-0001-5785-6799

Primary research

Keywords: Transthyretin, Amyloidosis, Familial, Saudi population, Epidemiology

DOl: https://doi.org/10.21203/rs.3.rs-386081/v1

License: (c) (i) This work is licensed under a Creative Commons Attribution 4.0 International License. Read Full License 


\section{Abstract}

\section{Background}

Familial transthyretin (TTR) amyloidosis (ATTR) is an autosomal dominant disease with significant phenotypic heterogeneity. Its prevalence in Saudi Arabia has not previously been investigated. An existing exome variant database of Saudi individuals, sequenced to globally investigate rare diseases in the population, was mined for TTR variants and filtered for missense mutations resulting in single amino acid changes. A total of 13,906 Saudi exomes from unrelated individuals were analyzed blindly.

Results

Three TTR variants known to be associated with ATTR amyloidosis were identified. Additionally, three novel TTR mutations were identified. Structural analysis of the three novel variants suggests that at least two could be amyloidogenic. The most common variant associated with amyloidosis was p.Val142lle (allele frequency 0.001). Further investigation of these variants and their translation to clinical practice may help to diagnose, monitor and manage patients with ATTR amyloidosis.

\section{Conclusion}

Multiple TTR variants potentially associated with systemic ATTR amyloidosis were identified in the Saudi population. Early diagnosis and intervention, facilitated by familial genetic testing of patients with ATTR amyloidosis, may benefit in the management of this disease. Early diagnosis could be enhanced through inclusion of ATTR variants in existing population-based screening programs.

\section{Background}

The tetrameric transthyretin (TTR) protein is stabilized by the binding of thyroid hormone or retinolbinding protein 4 (RBP4), preventing its dissociation into monomers that can aggregate into amyloid fibrils [1-3]. Pathogenic accumulation of amyloid TTR (ATTR) can be promoted either by misfolding of the wild-type protein, as seen in wild-type systemic amyloidosis, or by the presence of inherited or de novo mutations in the TTR gene on Hsa18 that result in single amino acid changes to the protein [4]. These changes may destabilize the tetramer by impeding binding to stabilizing thyroxine or RBP4. More than 120 hereditary TTR mutations that increase the rate of formation of amyloid fibrils have been identified $[5,6]$. Hereditary ATTR is an autosomal dominant disorder in which allelic heterogeneity impacts both penetrance and clinical manifestations [7]. Some variants are mostly associated with polyneuropathy, some can induce either early or late onset cardiomyopathy (CM), while for others, mixed phenotype (neuro and cardiological) may be present [7]. Phenotypes arising from the same variant can also differ between individuals due to several factors such as age, gender ethnicity and other unknown factors [8]. Polyneuropathy resulting from ATTR is progressive and involves autonomic and sensorimotor neural dysfunction that results in significant disability and death. Life expectancy of patients with hereditary ATTR ranges from 7 to 11 years after the diagnosis is established [9]. CM associated with ATTR (ATTR- 
$\mathrm{CM}$ ) tends to have a worse prognosis than polyneuropathy, with a life expectancy of 2 to 6 years following diagnosis [7].

Given the progressive nature of ATTR and the poor prognosis for patients with ATTR-CM and ATTRpolyneuropathy, early diagnosis is crucial to permit timely management. In clinical practice, a timely diagnosis remains difficult to achieve: symptomatic patients with amyloidosis experience an average delay of 4 years before a correct diagnosis is established $[10,11]$. A recent review recommended genetic screening of at-risk family members of patients with ATTR amyloidosis to identify pre-symptomatic individuals with pathogenic TTR variants, in order to allow early diagnosis and possibly early therapeutic intervention [12]. However, such an approach requires a good understanding of the prevalence of hereditary ATTR and of the clinical manifestations of TTR variants in the local population. At present, Arab populations are underrepresented in ATTR databases, thus limiting our ability to adopt such an approach in Saudi Arabia. Large-scale national genomic data mining can be very useful to establish frequency of pathological genetic variation resulting in rare diseases such as amyloidosis $[13,14]$. This type of study also offers the opportunity to identify novel variants that are potentially unique to the studied population and may have important epidemiologic, diagnostic and potentially therapeutic implications.

The objectives of this study are: 1) to analyze a national exome database in order to identify the incidence of known and candidate pathogenic variants associated with ATTR in Saudi Arabia, and 2) to investigate the amyloidogenic potential of novel candidate TTR variants.

\section{Results}

Analysis of the 13,906 Saudi exomes identified 180 TTR variants. Of these, 12 were missense mutations that were further investigated (Table 1). Eight variants were either known to be pathogenic or resulted in changes of unknown effect (Table 1). Five of the eight variants that were encountered in our study were previously reported. Of these, three variants are known to negatively impact function of the TTR protein, resulting in amyloidosis: c.239C > T(p.Thr80lle), c.238A > G(p.Thr80Ala) and c.424G > A(p.Val142lle) [5, 14-16]. The most frequent was c.424G $>A(p . V a l 142 \| l e)(0.001)$, whereas c.238A $>G(p . T h r 80 A l a)$ (0.00004) was the least common (Table 1). The remaining two previously reported variants, c.140A > $\mathrm{G}$ (p.Asn47Ser) and c.368G > A(p.Arg123His), have an unknown effect on TTR function [14]. Three novel TTR variants were identified by this study: c.404C > T (p.Ser135Phe), c.428C > T (p.Thr143lle) and c.298A $>\mathrm{G}$ (p.Lys100Glu), and were among the rarest in this cohort, with only 1 allele of each being observed (Table 1). 
Table 1

Identified missense TTR variants

\begin{tabular}{|c|c|c|c|}
\hline$T T R$ variant & Number of heterozygous & Number of homozygous & Allele frequency \\
\hline \multicolumn{4}{|c|}{ Known to affect TTR function } \\
\hline c.239C > T:p.Thr80lle & 2 & 0 & 0.00007191 \\
\hline c. 238 A > G:p.Thr80Ala & 1 & 0 & 0.00003596 \\
\hline c.424G > A:p.Val142lle & 28 & 0 & 0.00100676 \\
\hline \multicolumn{4}{|l|}{ Novel variants } \\
\hline c.404C > T:p.Ser135Phe & 1 & 0 & 0.00003596 \\
\hline c.428C > T:p.Thr143lle & 1 & 0 & 0.00003596 \\
\hline c.298A > G:p.Lys100Glu & 1 & 0 & 0.00003596 \\
\hline \multicolumn{4}{|c|}{ Variants of unknown significance } \\
\hline c.140A > G:p.Asn47Ser & 2 & 0 & 0.00007191 \\
\hline c.368G > A:p.Arg123His & 2 & 0 & 0.00007191 \\
\hline \multicolumn{4}{|l|}{ Does not affect function } \\
\hline c.76G > A:p.Gly26Ser & 178 & 0 & 0.00640012 \\
\hline c.328C > A:p.His110Asn & 239 & 6 & 0.00902488 \\
\hline c. $370 \mathrm{C}>$ T:p.Arg124Cys & 6 & 0 & 0.00021573 \\
\hline c.385G > A:p.Ala129Thr & 3 & 0 & 0.00010787 \\
\hline
\end{tabular}

Several high-resolution crystallographic structures exist for the TTR tetramer, in its apo-form, bound to stabilizing compounds, and bound to RBP4. One TTR tetramer binds to two RBP4 (Fig. 2A). One novel variant, c.298A > G (p.Lys100Glu) is located at the interface between two TTR molecules and RBP4 (Fig. 2B). Its replacement with an oppositely charged glutamic acid will alter the charge balance of this area, thus affecting association with RBP4. A second, c.404C > T (p.Ser135Phe) novel variant sits at the interface between the TTR tetramer and thyroxine. Replacing Ser135 with a much larger phenylalanine introduces steric clashes and leads to substantial changes in the thyroxine binding pocket because of the proximity of the four Ser135 from the four TTR tetramer chains (Fig. 2C). c.428C > T (p.Thr143lle) is located at the C-terminus of TTR. Its replacement with an isoleucine does not create steric hindrance and does not impact intra or intermolecular interactions. If a strong association of this variant with phenotype is established, then it may be related to other mechanisms, such as folding dynamics (Fig. 2D).

\section{Discussion}


This is the first study to identify and investigate the prevalence of pathogenic TTR variants in the population of Saudi Arabia, a population that is not represented in current databases. The investigation of TTR variants in a previously unstudied population would be expected to provide insights into the prevalence of known amyloidogenic variants and potentially identify novel variants that might be associated with systemic amyloidosis. Of the variants known to be associated with amyloidosis, c.424G $>A(p . V a l 142 l l e)$ was the most frequently identified in the Saudi population with an allele frequency of 0.001 , similar to the frequency reported in a multinational database [14]. This variant is most often detected in individuals of African descent, with approximately 3\% of African-Americans carrying at least one copy of the gene (allele frequency 0.0173 ) [17]. It is known to be associated with late onset $\mathrm{CM}$, more commonly in men (Table 2) [8, 17-20]. Another known amyloidogenic variant, c.238A > G(p.Thr80Ala), was found at a frequency of 0.00004 in our database, suggesting that it is rare in the Saudi population. This variant is relatively common in Ireland and the UK with $1.1 \%$ of the northwest Irish population being carriers $[15,16]$. It has also been found in other regions associated with Irish immigration, as well as in populations with no known Irish or UK ancestry [8]. This variant is associated with cardiac amyloidosis as well as autonomic and peripheral neuropathy (Table 2) [21, 22].

Table 2

Phenotypes of known amyloidogenic TTR variants with alleles identified in the Saudi population

\begin{tabular}{|c|c|}
\hline Variant & Phenotype \\
\hline \multirow{2}{*}{$\begin{array}{l}\text { c. } 424 G> \\
\text { A(p.Val142Ile) }[8,13, \\
18-20]\end{array}$} & - Late-onset cardiomyopathy (over the age of 65 ), more commonly in men \\
\hline & - Low amyloid load, remaining subclinical in many carriers \\
\hline \multirow{3}{*}{$\begin{array}{l}\text { c.238A > } \\
\text { G(p.Thr80Ala) }[8,15, \\
16,21,22]\end{array}$} & - Age of onset in seventh decade of life \\
\hline & - Cardiac amyloidosis and autonomic and peripheral neuropathy \\
\hline & $\begin{array}{l}\text { - Gastrointestinal disorders are common. Carpal tunnel syndrome precedes } \\
\text { other symptoms in nearly three quarters of patients }\end{array}$ \\
\hline \multirow{2}{*}{$\begin{array}{l}\text { c. } 239 \mathrm{C}>\mathrm{T}(\mathrm{p} . \mathrm{Thr} 80 \mathrm{lle}) \\
\text { [5] }\end{array}$} & - Likely pathogenic, but there are no reports of the clinical manifestation \\
\hline & $\begin{array}{l}\text { - It is similar to } p \text {.Thr80Ala, as both substitute a native uncharged polar } \\
\text { residue with a nonpolar residue }\end{array}$ \\
\hline
\end{tabular}

Our study also identified two individuals with another known amyloidogenic variant, c.239C > $T$ (p.Thr80lle), with an allele frequency of 0.00007 . There are few published reports of this variant, suggesting that it is rare. The phenotype associated with this variant has not been previously reported in the literature. It is absent in major databases (1000 Genomes Project, Exome Variant Server, Genome Aggregation Database), consistent with it being ethnically restricted. However, it is recorded in the Mutations in Hereditary Amyloidosis database as being amyloidogenic [5] and its similarity to the p.Thr80Ala variant suggests that its clinical implications might be predicted (Table 2). Interestingly, some of the most common variants identified in other populations are not observed in the Saudi database of 13,906 individuals. These include: $c .148 \mathrm{G}>\mathrm{A}(\mathrm{p}$.Val50Met), the most common variant in Western Europe [8]; p.Leu131Met, a variant with cardiac manifestations predominantly found in Denmark [8, 23]; 
p.lle88Leu, which is seen predominantly in the Italian population $[8,24]$ and has primarily cardiac manifestations [24]; p.Val50Ala, p.Ala117Ser and p.Gly103Arg, the most common amyloidogenic variants in the Chinese population [25]; and p.Ser70Arg, the most common variant in Mexico [26]. The absence of these variants from our database does not imply that they are absent in the Saudi population. However, if present, they are likely to have a very rare prevalence. Our findings further indicate that there is significant population variation in the prevalence of TTR variants, including some variants that are relatively restricted to certain ethnic groups. The fact that some of the TTR variants identified in the Saudi database have not been previously reported suggests that they might be restricted to the Saudi and possibly neighboring populations. Two of the three novel variants in our study were predicted by computational structural analysis to result in a reduced capacity of the TTR protein to associate with thyroid hormone or RBP4. These associations are thought to aid in the stabilization of the TTR tetramer and prevent it from dissociating into fibril-forming monomer aggregates [1-3]. Thus, it is possible that patients carrying these variations are at risk of developing future clinical amyloidosis. However, this in silico analysis may be considered as only "predictive" and further investigations are required to confirm the clinical implications of these novel variants.

The study is limited by sample size $(13,906)$ which, while relatively large, will not identify very rare or de novo ATTR alleles within the population. The use of a cohort of 13,906 unrelated individuals, primarily sequenced to investigate rare inherited diseases globally, is not expected to introduce any bias in the ascertainment of allele frequencies. Individuals represented in our database originate from a large geographic area encompassing different regions within Saudi Arabia, thus allowing adequate estimation of $T T R$ variants that are not vanishingly rare in the country $[27,28]$. Another limitation of our study is that the interpretation of the clinical implication of novel pathogenic variants is restricted, as there is little clinical information and follow-up linked to the exome data. While one may predict that at least two of the three novel variants identified in our database are potentially amyloidogenic using structural analysis, without family histories and segregation, one cannot assume that such variants will ultimately result in clinical ATTR amyloidosis. This limitation also applies to variants of known function. How these data link to clinical manifestations in the population of Saudi Arabia remains unknown and, therefore, no comparison of penetrance or manifestations of identified variants between Saudi and other studied populations can be made. Such information could provide a valuable insight on how genetic background may influence the penetrance and manifestations of TTR variants. Furthermore, the prevalence of identified variants cannot be compared to the number of patients with a known diagnosis, precluding any attempts to determine if there is significant underdiagnosis in Saudi Arabia. Studies in other populations have noted the potential for extensive underdiagnosis $[29,30]$.

The data described in this manuscript suggest that there are TTR variants potentially associated with amyloidosis in Saudi Arabia and highlight the need for further clinical data regarding this patient population. Reports from other populations suggest that a concerted effort is required to identify, monitor and manage individuals with pathogenic TTR variants. This approach would likely allow therapeutic intervention before considerable deposition of amyloid fibrils induces symptoms and advanced organ damage $[12,31]$. In Saudi Arabia, such efforts could focus on: the routine genetic testing of patients with 
phenotypes that raise suspicion of amyloidosis irrespective of age; the development of genetic testing programs for relatives of patients with known ATTR amyloidosis and the utilization of existing newborn and pre-marital genetic screening programs to identify carriers of pathogenic TTR variants; the development of a consensus on optimal monitoring and management of patients with pathogenic TTR variants, including appropriate genetic counseling for family members; and finally, establishing a registry for patients with amyloidosis. In individuals with a phenotype suspicious for amyloidosis such as hypertrophic cardiomyopathy, the presence of a TTR mutation has diagnostic implications and points away from a myocardial sarcomeric disease and towards amyloidosis. The identification of potentially pathogenic TTR mutations has important clinical implications for the classification, diagnosis, and treatment of amyloidosis. Within the disease context, the detection of a mutation allows one to classify TTR amyloidosis as hereditary rather than wild-type and should prompt consideration for genetic screening of siblings. Genetic screening conducted on siblings of patients with the hereditary form allows for the detection of mutation carriers who are at risk of developing future clinical amyloidosis. Saudi Arabia benefits from extensive existing screening programs, meaning that there is infrastructure already in place to facilitate screening for TTR variants in people known to be at risk and in the wider population.

The aim of these screening programs is to identify patients before they become symptomatic. A recent article by Conceição and colleagues [12] on early diagnosis and follow-up cautioned that it is important to minimize any anxiety that could be caused through over-medicalization from the knowledge of carrier status and repeated follow-up. In order to determine how these patients should be monitored in Saudi Arabia, it is therefore important to carefully consider and gain consensus on when patients should begin to be monitored and at what time intervals; which clinicians and other healthcare professionals should be involved; what should be done for patients with TTR variants of currently uncertain effect; when treatment should be initiated; and which tests should be performed to monitor patients. In this context, biomarkers such as serum TTR levels or urine RBP4 [32] have potential for more widespread use in the clinic. Furthermore, current imaging tools can allow the detection of organ involvement at a subclinical stage, before overt morphological abnormalities become evident. For example, cardiac imaging using Tclabelled phosphate compounds with high affinity for TTR allows early diagnosis of amyloid myocardial deposition, not only at a pre-symptomatic stage, but also before an increase in wall thickness is detected by echocardiography and prior to the development of electrocardiographic abnormalities [33]. A recently published Japanese expert opinion recommended periodic clinical assessment as well as various investigations in monitoring asymptomatic gene mutation carriers [31]. With the advent of drugs that help stabilize the TTR tetramer, reduce tissue deposition and possibly slow disease progression, [34] an early diagnosis guided by genetic screening should be strongly pursued. Such drugs may well prove to be more effective if administered before the development of overt tissue involvement and organ damage. Figure 3 illustrates a framework for the initial assessment and follow-up of individuals who are asymptomatic carriers of TTR mutations.

\section{Conclusion}


We present data to suggest that the Saudi population has a unique subset of TTR variants and possibly lacks variants commonly present in other patient populations. Additionally, we identified the presence of novel, potentially pathogenic, TTR variants. Our findings support the need for a nationwide registry and a framework for genetic testing with systematic patient evaluation and follow-up.

\section{Methods}

\section{TTR variant data mining and filtration}

The database comprises 13,906 consecutive exomes from unrelated Arab individuals, sequenced between 2015 and 2019 as part of a global investigation of rare diseases in the Saudi population [27, 28, 35]. To the best of our knowledge, no sample biases likely to enrich or deplete TTR variants are present. All TTR (NM_000371) variants were selected from the database for analysis and were further filtered to include non-synonymous and potentially pathogenic variants (Fig. 1). Synonymous variants and changes known to have no pathogenic effect were removed. Since ATTR results from misfolded rather than absent or truncated protein, splice variants, stop codons or frameshift mutations were also excluded. The remaining variants were classified as pathogenic, likely pathogenic, or variants of uncertain significance according to recommendations published by the American College of Medical Genetics [36]. We used available databases and published data to identify variants associated with clinical ATTR amyloidosis in other patients. The following publicly available sources were used: ClinVar (www.ncbi.nlm.nih.gov/clinvar/), the Mutations in Hereditary Amyloidosis database (www. amyloidosismutations.com), the Human Gene Mutation database (www.hgmd.cf.ac.uk) and literature from PubMed (www.PubMed.com).

\section{Computational structural analysis of novel mutations}

Novel variants were characterized with computational structural analysis that used thyroxin-bound TTR (PDB ID: 2rox) and RBP-bound TTR (PDB ID: 2wqa) as a reference, to identify and analyze the structural and functional repercussions associated with each variant. The PyMOL program (pymol.org) was used to inspect the models obtained for each variant.

\section{Declarations}

\section{Ethics statement}

The present study was performed according to the principles of the Declaration of Helsinki and the legal requirements of Saudi Arabia

\section{Consent for publication}

Not applicable. 


\section{Data availability statement}

Due to the nature of this research, participants of this study did not agree for their data to be shared publicly, so supporting data is not available.

\section{Competing interests}

The authors declare no conflicts of interest regarding the production of this article. The authors have no personal financial or institutional interest in any of the drugs, materials, or devices described in this article.

\section{Funding details}

The structural analysis by Stefan T. Arold reported in this publication was supported by funding from King Abdullah University of Science and Technology (KAUST) through the baseline fund and the Award No. FCC1/1976-25 from the Office of Sponsored Research.

\section{Author Contribution}

1. Abouelhoda - conception and design; acquisition and analysis of data

2. Mohty - acquisition and analysis of data; drafting the manuscript

3. Alayary - acquisition and analysis of data; drafting the manuscript

4. Meyer - conception and design, acquisition and analysis of data; drafting the manuscript

5. Arold - acquisition and analysis of data; drafting the manuscript

6. Fadel - acquisition and analysis of data; drafting the manuscript and figures

7. Monies - conception and design; acquisition and analysis of data; drafting the manuscript and figures

\section{Acknowledgements}

Editorial assistance was provided by Abigail Killen-Devine and Kyle Lambe of Synergy Medical Communications UK.

\section{References}

1. Miroy GJ, Lai Z, Lashuel HA, Peterson SA, Strang C, Kelly JW: Inhibiting transthyretin amyloid fibril formation via protein stabilization. Proc Natl Acad Sci U S A 1996, 93(26):15051-15056.

2. Rostom AA, Sunde M, Richardson SJ, Schreiber G, Jarvis S, Bateman R, Dobson CM, Robinson CV: Dissection of multi-protein complexes using mass spectrometry: Subunit interactions in transthyretin and retinol-binding protein complexes. 1998, 33(S2):3-11.

3. White JT, Kelly JW: Support for the multigenic hypothesis of amyloidosis: The binding stoichiometry of retinol-binding protein, vitamin $\mathrm{A}$, and thyroid hormone influences transthyretin amyloidogenicity 
\&lt;em\&gt;in\&lt;/em\&gt; \&lt;em\&gt;vitro\&lt;/em\&gt. Proceedings of the National Academy of Sciences 2001, 98(23):13019.

4. Ruberg FL, Grogan M, Hanna M, Kelly JW, Maurer MS: Transthyretin Amyloid Cardiomyopathy: JACC State-of-the-Art Review. Journal of the American College of Cardiology 2019, 73(22):2872-2891.

5. Rowczenio DM, Noor I, Gillmore JD, Lachmann HJ, Whelan C, Hawkins PN, Obici L, Westermark P, Grateau G, Wechalekar AD: Online registry for mutations in hereditary amyloidosis including nomenclature recommendations. Human mutation 2014, 35(9):E2403-2412.

6. Yee AW, Aldeghi M, Blakeley MP, Ostermann A, Mas PJ, Moulin M, de Sanctis D, Bowler MW, MuellerDieckmann C, Mitchell EP et al: A molecular mechanism for transthyretin amyloidogenesis. Nat Commun 2019, 10(1):925-925.

7. Maurer MS, Bokhari S, Damy T, Dorbala S, Drachman BM, Fontana M, Grogan M, Kristen AV, Lousada I, Nativi-Nicolau J et al: Expert Consensus Recommendations for the Suspicion and Diagnosis of Transthyretin Cardiac Amyloidosis. Circulation Heart failure 2019, 12(9):e006075.

8. Damy T, Kristen AV, Suhr OB, Maurer MS, Planté-Bordeneuve V, Yu CR, Ong ML, Coelho T, Rapezzi C: Transthyretin cardiac amyloidosis in continental Western Europe: an insight through the Transthyretin Amyloidosis Outcomes Survey (THAOS). European heart journal 2019.

9. Parman Y, Adams D, Obici L, Galán L, Guergueltcheva V, Suhr OB, Coelho T: Sixty years of transthyretin familial amyloid polyneuropathy (TTR-FAP) in Europe: where are we now? A European network approach to defining the epidemiology and management patterns for TTR-FAP. Current opinion in neurology 2016, 29 Suppl 1(Suppl 1):S3-s13.

10. McCausland KL, White MK, Guthrie SD, Quock T, Finkel M, Lousada I, Bayliss MS: Light Chain (AL) Amyloidosis: The Journey to Diagnosis. The patient 2018, 11(2):207-216.

11. Hawkins PN, Ando Y, Dispenzeri A, Gonzalez-Duarte A, Adams D, Suhr OB: Evolving landscape in the management of transthyretin amyloidosis. Ann Med 2015, 47(8):625-638.

12. Conceição I, Damy T, Romero M, Galán L, Attarian S, Luigetti M, Sadeh M, Sarafov S, Tournev I, Ueda M: Early diagnosis of ATTR amyloidosis through targeted follow-up of identified carriers of TTR gene mutations. Amyloid : the international journal of experimental and clinical investigation : the official journal of the International Society of Amyloidosis 2019, 26(1):3-9.

13. Damrauer SM, Chaudhary K, Cho JH, Liang LW, Argulian E, Chan L, Dobbyn A, Guerraty MA, Judy R, Kay J et al: Association of the V122I Hereditary Transthyretin Amyloidosis Genetic Variant With Heart Failure Among Individuals of African or Hispanic/Latino Ancestry. Jama 2019, 322(22):2191-2202.

14. Lahuerta Pueyo C, Aibar Arregui M, Gracia Gutierrez A, Bueno Juana E, Menao Guillén S: Estimating the prevalence of allelic variants in the transthyretin gene by analysing large-scale sequencing data. European journal of human genetics : EJHG 2019, 27(5):783-791.

15. Carr AS, Pelayo-Negro AL, Evans MR, Laurà M, Blake J, Stancanelli C, lodice V, Wechalekar AD, Whelan CJ, Gillmore JD et al: A study of the neuropathy associated with transthyretin amyloidosis (ATTR) in the UK. Journal of neurology, neurosurgery, and psychiatry 2016, 87(6):620-627. 
16. Reilly MM, Staunton H, Harding AE: Familial amyloid polyneuropathy (TTR ala 60) in north west Ireland: a clinical, genetic, and epidemiological study. Journal of neurology, neurosurgery, and psychiatry 1995, 59(1):45-49.

17. Jacobson DR, Alexander AA, Tagoe C, Buxbaum JN: Prevalence of the amyloidogenic transthyretin (TTR) V122I allele in 14333 African-Americans. Amyloid : the international journal of experimental and clinical investigation : the official journal of the International Society of Amyloidosis 2015, 22(3):171-174.

18. Buxbaum J, Alexander A, Koziol J, Tagoe C, Fox E, Kitzman D: Significance of the amyloidogenic transthyretin Val 122 lle allele in African Americans in the Arteriosclerosis Risk in Communities (ARIC) and Cardiovascular Health (CHS) Studies. American heart journal 2010, 159(5):864-870.

19. Jacobson DR, Pastore RD, Yaghoubian R, Kane I, Gallo G, Buck FS, Buxbaum JN: Variant-sequence transthyretin (isoleucine 122) in late-onset cardiac amyloidosis in black Americans. The New England journal of medicine 1997, 336(7):466-473.

20. Quarta CC, Buxbaum JN, Shah AM, Falk RH, Claggett B, Kitzman DW, Mosley TH, Butler KR, Boerwinkle E, Solomon SD: The amyloidogenic V122I transthyretin variant in elderly black Americans. The New England journal of medicine 2015, 372(1):21-29.

21. Sattianayagam PT, Hahn AF, Whelan CJ, Gibbs SD, Pinney JH, Stangou AJ, Rowczenio D, Pflugfelder PW, Fox Z, Lachmann HJ et al: Cardiac phenotype and clinical outcome of familial amyloid polyneuropathy associated with transthyretin alanine 60 variant. European heart journal 2012, 33(9):1120-1127.

22. Wallace MR, Dwulet FE, Conneally PM, Benson MD: Biochemical and molecular genetic characterization of a new variant prealbumin associated with hereditary amyloidosis. $J$ Clin Invest 1986, 78(1):6-12.

23. Suhr OB, Svendsen IH, Andersson R, Danielsson $\AA$, Holmgren G, Ranløv PJ: Hereditary transthyretin amyloidosis from a Scandinavian perspective. 2003, 254(3):225-235.

24. Gagliardi C, Perfetto F, Lorenzini M, Ferlini A, Salvi F, Milandri A, Quarta CC, Taborchi G, Bartolini S, Frusconi S et al: Phenotypic profile of lle68Leu transthyretin amyloidosis: an underdiagnosed cause of heart failure. European journal of heart failure 2018, 20(10):1417-1425.

25. Yin J, Xia X, Shi Y, Lu Y, Zhao C, Huang Z, Tian N: Chinese familial transthyretin amyloidosis with vitreous involvement is associated with the transthyretin mutation Gly83Arg: a case report and literature review. Amyloid : the international journal of experimental and clinical investigation : the official journal of the International Society of Amyloidosis 2014, 21(2):140-142.

26. González-Duarte A, Cárdenas-Soto K, Bañuelos CE, Fueyo O, Dominguez C, Torres B, Cantú-Brito C: Amyloidosis due to TTR mutations in Mexico with 4 distincts genotypes in the index cases. Orphanet Journal of Rare Diseases 2018, 13(1):107.

27. Abouelhoda M, Faquih T, El-Kalioby M, Alkuraya FS: Revisiting the morbid genome of Mendelian disorders. Genome Biology 2016, 17(1):235. 
28. Abouelhoda M, Sobahy T, El-Kalioby M, Patel N, Shamseldin H, Monies D, Al-Tassan N, Ramzan K, Imtiaz F, Shaheen $\mathrm{R}$ et al: Clinical genomics can facilitate countrywide estimation of autosomal recessive disease burden. Genetics in medicine : official journal of the American College of Medical Genetics 2016, 18(12):1244-1249.

29. Damy T, Costes B, Hagège AA, Donal E, Eicher JC, Slama M, Guellich A, Rappeneau S, Gueffet JP, Logeart $\mathrm{D}$ et al: Prevalence and clinical phenotype of hereditary transthyretin amyloid cardiomyopathy in patients with increased left ventricular wall thickness. European heart journal 2016, 37(23):1826-1834.

30. Schmidt HH, Waddington-Cruz M, Botteman MF, Carter JA, Chopra AS, Hopps M, Stewart M, Fallet S, Amass $\mathrm{L}$ : Estimating the global prevalence of transthyretin familial amyloid polyneuropathy. Muscle \& nerve 2018, 57(5):829-837.

31. Ueda M, Sekijima Y, Koike H, Yamashita T, Yoshinaga T, Ishii T, Ando Y: Monitoring of asymptomatic family members at risk of hereditary transthyretin amyloidosis for early intervention with diseasemodifying therapies. Journal of the Neurological Sciences 2020, 414.

32. Hendren NS, Roth LR, Grodin JL: Disease-Specific Biomarkers in Transthyretin Cardiac Amyloidosis. Current heart failure reports 2020, 17(3):77-83.

33. Rapezzi C, Quarta CC, Guidalotti PL, Pettinato C, Fanti S, Leone O, Ferlini A, Longhi S, Lorenzini M, Reggiani LB et al: Role of $(99 \mathrm{~m})$ TC-DPD scintigraphy in diagnosis and prognosis of hereditary transthyretin-related cardiac amyloidosis. JACC Cardiovascular imaging 2011, 4(6):659-670.

34. Teng C, Li P, Bae JY, Pan S, Dixon RAF, Liu Q: Diagnosis and treatment of transthyretin-related amyloidosis cardiomyopathy. $\mathrm{n} / \mathrm{a}(\mathrm{n} / \mathrm{a})$.

35. Saudi Mendeliome G: Comprehensive gene panels provide advantages over clinical exome sequencing for Mendelian diseases. Genome Biology 2015, 16(1):134.

36. Richards S, Aziz N, Bale S, Bick D, Das S, Gastier-Foster J, Grody WW, Hegde M, Lyon E, Spector E et al: Standards and guidelines for the interpretation of sequence variants: a joint consensus recommendation of the American College of Medical Genetics and Genomics and the Association for Molecular Pathology. Genetics in medicine : official journal of the American College of Medical Genetics 2015, 17(5):405-424.

\section{Figures}




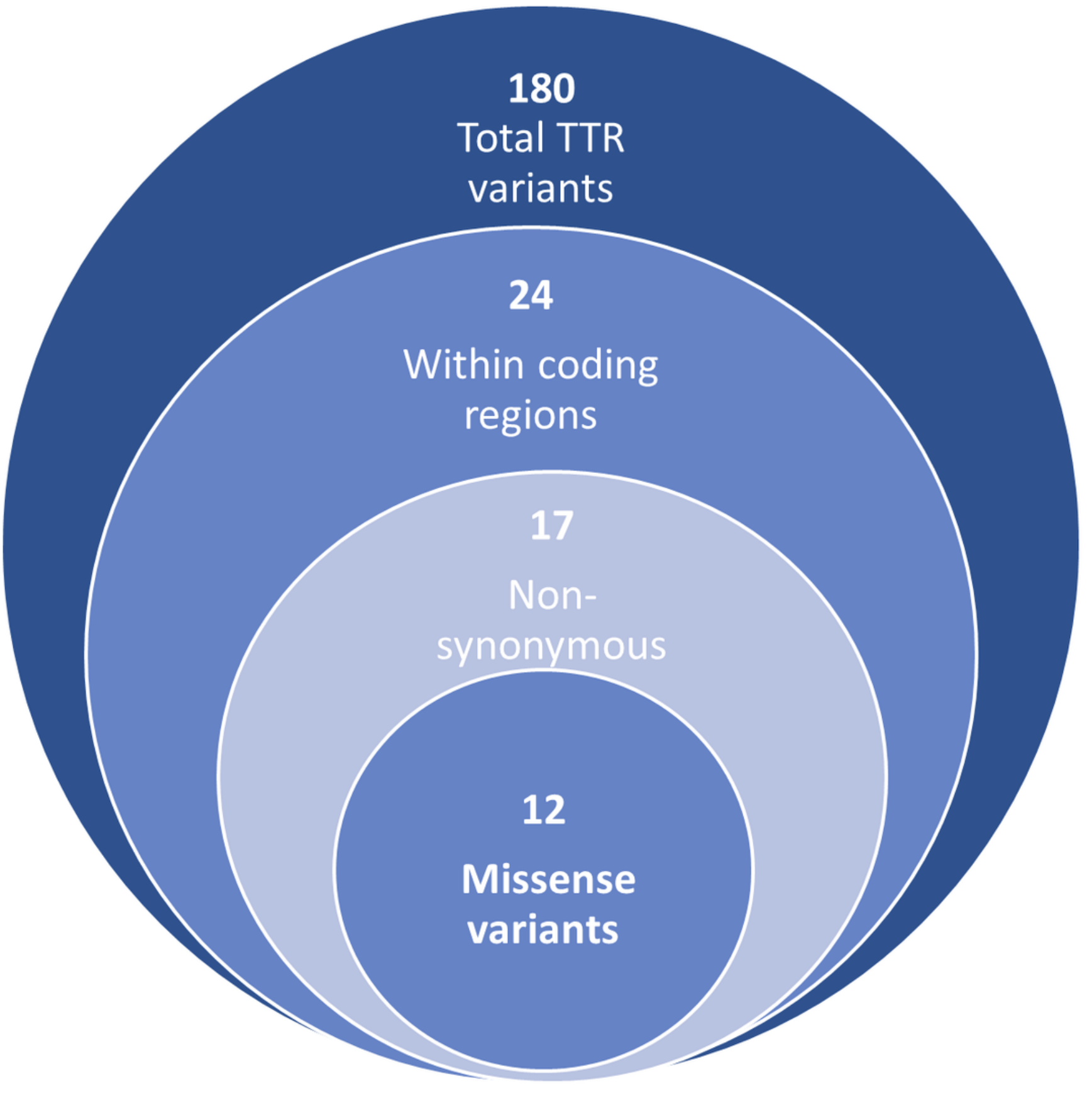

Figure 1

Schematic Representation of the Filtration Process 

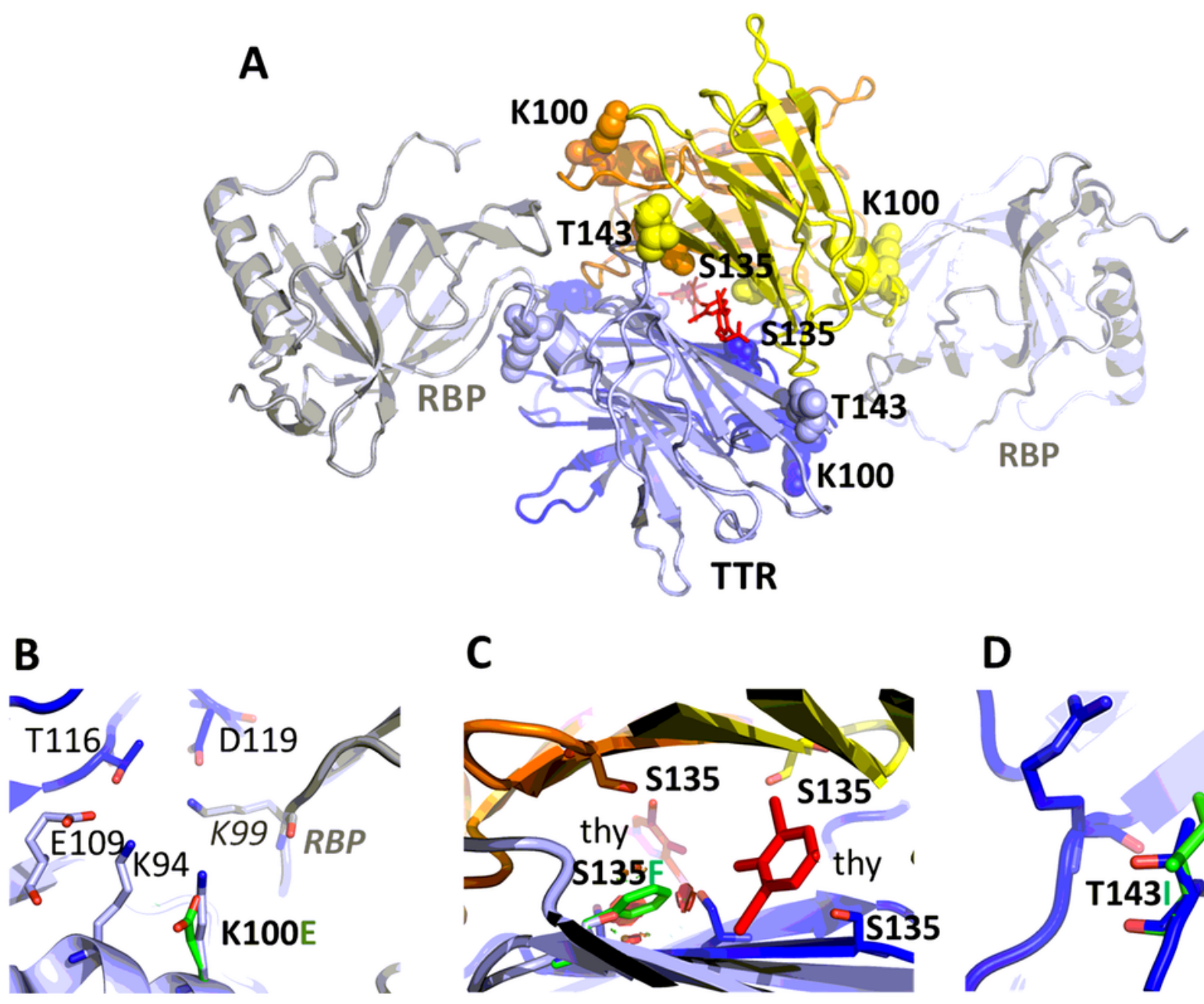

C

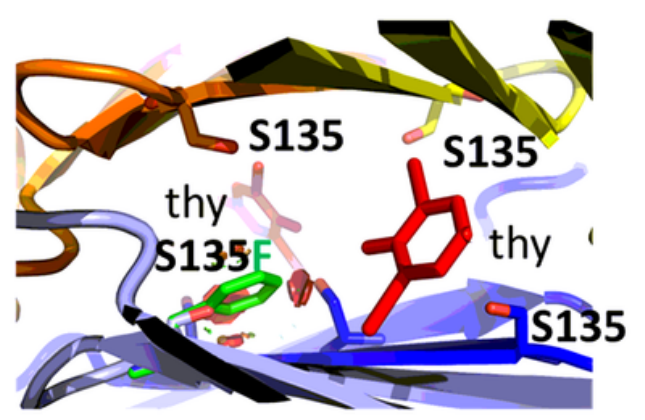

D

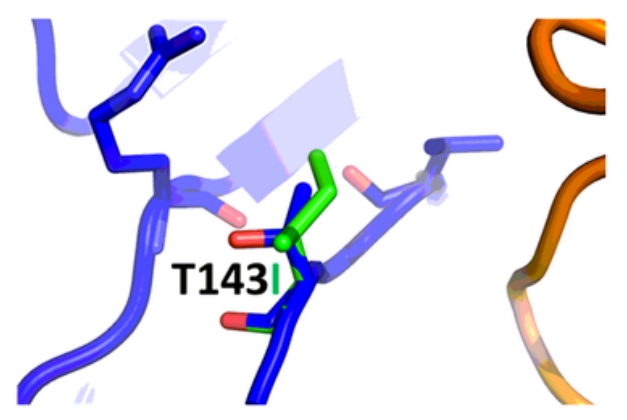

Figure 2

Computational structural analysis of novel variants. Mapping of the mutations onto the TTR tetramer. A) The TTR tetramer (each chain is color-coded) bound to RBP (gray) and thyroxin (thy, red). The mutated residues are shown as sphere models, corresponding to the frames with the zoomed-in regions. For clarity, not all residues are labelled. Note that the RBP binding environment of the same residue is different in different TRR chains. Figures B-D show the local environment of each mutated residue. The side chains of the variants are shown in green. In $C$, the red spheres illustrate the steric clashes resulting from the mutation. 


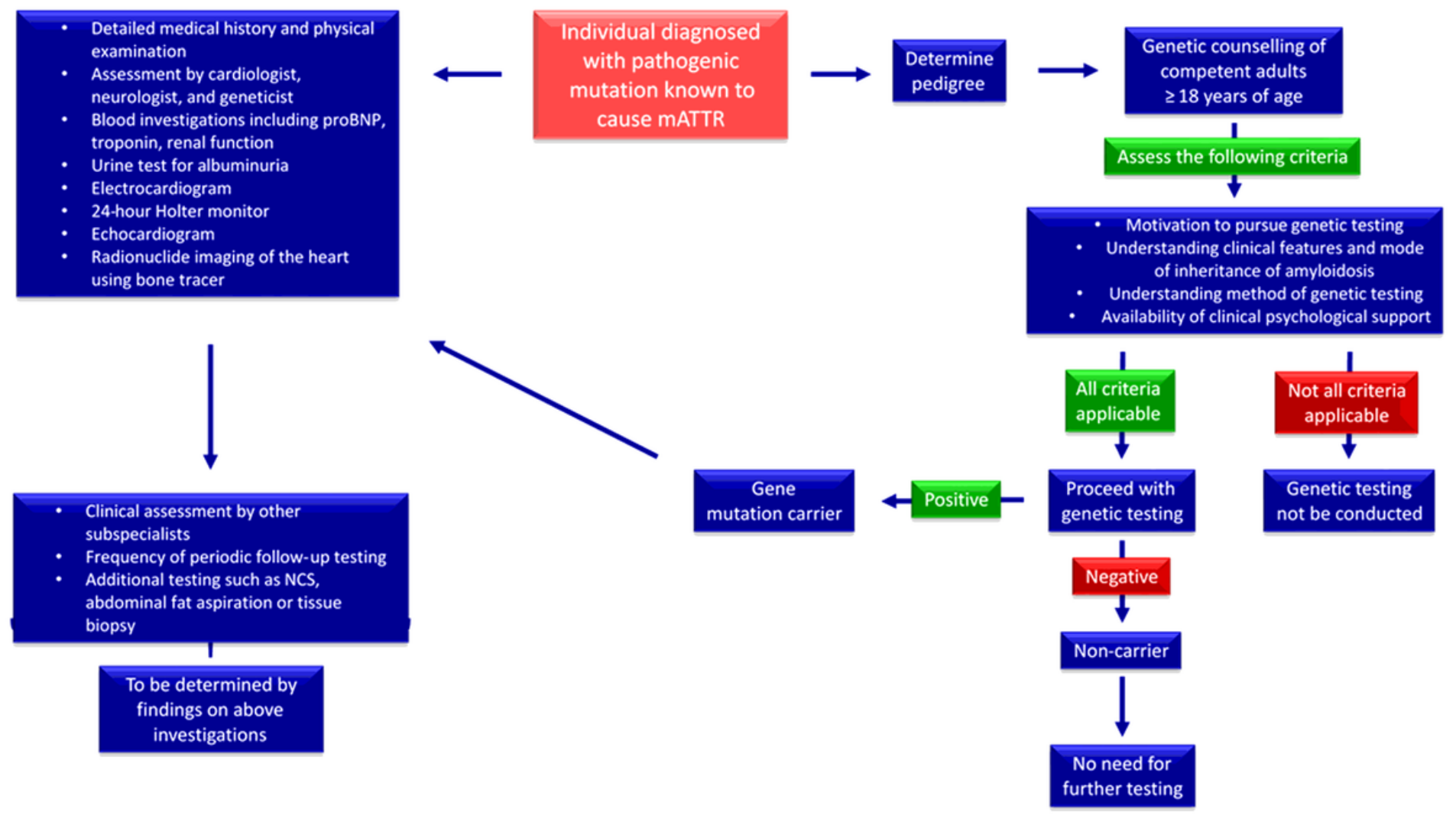

Figure 3

Framework for initial assessment and follow-up of asymptomatic carriers of TTR mutations. 